\title{
SOME THEOREMS ON FACTORIZATION OF MEROMORPHIC FUNCTIONS
}

\author{
BY FRED GROSS
}

Communicated by M. Gerstenhaber, March 13, 1968

In [3] the author proved

THEOREM 1. ${ }^{1}$ If $f$ is any entire function of lower order less than $\frac{1}{2}$ and $g$ is entire, then $f(g)$ is periodic if and only if $g$ is.

By means of a result due to Edrei [1] and Ostrovskii [6] it is possible to generalize Theorem 1 to a certain class of meromorphic functions. We begin with

LeMma 1 (EDReI [1], OstrovsKII [6]). Let $f(z)$ be meromorphic of lower order $\lambda<\frac{1}{2}$. If $\delta(\infty, f)>1-\cos \pi \lambda$, then $\left|f\left(\mathrm{re}^{i \theta}\right)\right| \rightarrow \infty$, uniformly in $\theta$ as $r_{n} \rightarrow \infty$ through a suitable sequence.

Here $\delta$ is the Nevanlinna deficiency (see Hayman [5, p. 42]).

THEOREM 2. Let $f$ be meromorphic of lower order $\lambda$ and let $g$ be entire. If $0 \leqq \lambda<\frac{1}{2}$ and for some $a, \delta(a, f)>1-\cos \pi \lambda$, then $f(g)$ is periodic if and only if $g$ is. If $\tau$ is a period of $f(g)$, then $g$ has a period having the same argument as $\tau$.

Sketch of Proof. We assume that $f(g)$ is periodic with period $\tau$ having argument $\alpha$. Let $L$ be the half line re ${ }^{i \alpha}$ everywhere except near poles of $f(g)$, where we let $L$ loop around them with radius $\epsilon, \epsilon$ a small positive number. Letting $f^{*}(z)=1 /(f(z)-a)$ and applying Lemma 1 we see that $\left|f^{*}\left(\mathrm{re}^{i \theta}\right)\right| \rightarrow \infty$, uniformly in $\theta$ as $r_{n} \rightarrow \infty$ through a suitable sequence. From the hypotheses of the theorem it follows that $f(g)$ is bounded on $L$. If $g$ is bounded on $L$, then as in the proof of Theorem 1 (see [3]) $g$ must be periodic with a period having the same argument as $\tau$. If $g$ is unbounded on $L$, then $f$ is bounded on $g(L)$ and this leads to a contradiction via Lemma 1.

CoRollary. If $P$ is a polynomial and $f$ is as in Theorem 2 , then $f(P)$ is not periodic.

This Corollary is a partial solution to the more general question suggested in [4]: If $f$ is meromorphic for which polynomials is $f(P)$ periodic?

${ }^{1} \mathrm{~N}$. Baker proved an analogue of this theorem for $f$ of order $<1 / 2$. See On some results of $A$. Renyi and $C$. Renyi concerning periodic entire functions, Acta Sci. Math. (Szeged) 27 (1966), 197-200. 
Theorem 2 also yields a generalization of an earlier result mentioned in [4].

THEOREM 3. Let $f$ be meromorphic and $g$ entire. If $f(g)$ is of finite order, has no deficient values and is periodic, with period $\tau$, then either $f$ has no deficient values or $g$ is periodic with a period having the same argument as $\tau$.

Sketch of Proof. By a theorem of Edrei and Fuchs [2] either $f$ is of zero order or $g$ is a polynomial. In the latter case $f$ can certainly not have any deficient values since $f(g)$ does not. In the former case one can apply Theorem 2 and arrive at the desired conclusion.

Corollary (SeE [4]). Let $f$ be meromorphic and $g$ entire. If $f(g)$ is elliptic, then $f$ has no deficient values.

This last corollary rules out the possibility of applying the earlier one to resolve the question mentioned in [4]: If $P$ is a polynomial of degree $n$, where $n=5$ or $n \geqq 7$ and $f$ is any meromorphic function, then $f(g)$ is not elliptic?

\section{REFERENCES}

1. A. Edrei, The deficiences of functions of finite lower order, Duke Math. J. 31 (1964), 1-22.

2. A. Edrei and W. H. Fuchs, On the zeros of $f(g(z))$ where $f$ and $g$ are entire functions, J. Analyse Math. 12 (1964), 243-255.

3. F. Gross, On factorization of meromorphic functions, Trans Amer. Math. Soc. 131 (1968), 215-221.

4. - On factorization of elliptic functions, Canad. J. Math. 20 (1968), 486-494.

5. W. K. Hayman, Meromorphic functions, Oxford Mathematical Monographs, Clarendon Press, Oxford, 1964.

6. I. V. Ostrovskii, On the deficiencies of meromorphic functions of lower order less than one, Dokl. Akad. Nauk SSSR 150 (1963), 32-35= Soviet Math. Dokl. 4 (1963), 587-591.

Bellcomm Incorporated, Washington, D. C. 